\title{
The Stabilizing Effect of a Magnetic Field on the Plasma Production of a Vacuum-Discharge Rotating Plasma Gun
}

\author{
Member Takashi Ikehata \\ (Ibaraki University) \\ Non-member Kazuyuki Hasegawa (Ibaraki University) \\ Non-member Toshio Tanabe \\ (Ibaraki University) \\ Non-member Naoyuki Sato \\ (Ibaraki University) \\ Member Hiroshi Mase \\ (Ibaraki University)
}

\begin{abstract}
A coaxial plasma gun which generates a rapidly rotating plasma in a magnetic field has been developed to improve the plasma centrifuge. It is demonstrated that a magnetic field stronger than a critical value is essential to stabilize the high-current vacuum discharge of the plasma gun and to make the plasma production and acceleration reproducible. For example, the critical field is $250 \mathrm{G}$ for the discharge current of $13 \mathrm{kA}$ and the anode-cathode spacing of $7.5 \mathrm{~mm}$. A model is presented in which the critical field is assumed to be determined from the trapping of ionizing electrons in the annular anode-cathode gap. A qualitative agreement is achieved between the model and experiment.
\end{abstract}

Key words : Plasma centrifuge, Isotope separation, Plasma production, Electric discharges, High vacuum, Magnetic fields, Stability, Electron beams, Orbits

\section{Introduction}

Plasmas of metal elements have been used for many applications such as ion implantation and ion beam modification of materials ${ }^{(1)}$, thin-film deposition ${ }^{(2)}$, heavy ion beam fusion ${ }^{(3)}$, and plasma isotope separation ${ }^{(4) \sim(6)}$. In recent years, a vacuum discharge has received an interest as a meants of producing metal plasmas, by reasons that the apparatus is simple and easy to construct; in addition, a number of solid elements and their alloys and compounds are available only by changing a cathode material ${ }^{(7)}$. A problem has also been pointed out, however, that the vacuum discharge tends to become unstable ${ }^{(8)}$ : Cathode spots and current spokes often appear to move about on the electrode and in the glow region, respectively. This leads to the fluctuation in the voltage and plasma density. This result is attributable to the fact that the metal vapor as the source of plasma is provided by the vacuum discharge itself.

We have developed a coaxial, rotating plasma gun to explore the isotope separation by the plasma centrifuge, where rotating $\mathrm{Cu}, \mathrm{Cu} / \mathrm{Zu}, \mathrm{Al}, \mathrm{Ag}$ and $\mathrm{Ta}$ plasmas of densities $10^{14} \sim 10^{15} \mathrm{~cm}^{-3}$ and rotation frequencies $10^{5}$ $\sim 10^{6} \mathrm{rad} / \mathrm{s}$ have been produced by the vacuum discharge under a magnetic field of up to $3 \mathrm{kG}^{(9)}$. The present paper reports that a magnetic field is essential not only to drive the plasma rotation but also to stabilize the vacuum discharge.

\section{Experimental apparatus}

Experimental arrangement is shown in Fig. 1. The plasma gun has a coaxial geometry where a rod cathode ( $\phi 3 \sim 8 \mathrm{~mm} \times 40 \mathrm{~mm}$ ) which provides the metal vapor is placed inside the annular brass anode $(\phi 25 \mathrm{~mm})$. The main vacuum discharge between the anode and cathode is triggered by a $8 \sim 10 \mathrm{kV}$ impulse spark on a tungsten tip $(\phi 0.5 \mathrm{~mm})$ inserted into the small bore of the cathode and is energized by a $12 \mathrm{kV}, 40 \mu \mathrm{F}$ capacitor bank. The plasma gun is attached to the end flange of the chamber where an axial magnetic field $B_{z}$ of up to 3.0 $\mathrm{kG}$ is applied over the length of $40 \mathrm{~cm}$. The voltage and current of the discharge are measured with a resistive divider and a Rogowski coil, respectively. The rotation frequency $\omega$ and the ion temperature $T_{i}$ of the plasma are evaluated from doppler shift and doppler broadening of ion emission lines. An optical multichannel analyzer (OMA) which combines a MCP-intensified, 1,024channel photodiode array with a $1 \mathrm{~m}$ spectrometer is 


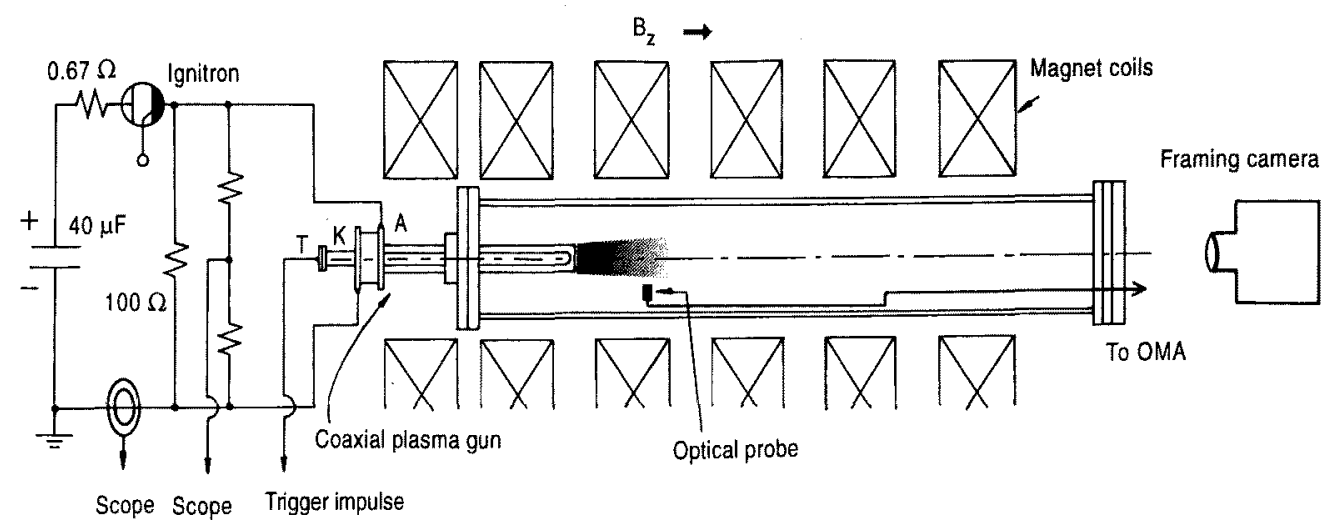

Fig. 1. Experimental setup.

used for this purpose. This system records the wavelength range of $20 \mathrm{~nm}$ with the resolution of $0.02 \mathrm{~nm}$ per channel by a single exposure. The light from the plasma is focused by a small lens $(\phi 1.8 \mathrm{~mm})$ to the end of a fiber cable and is transmitted to OMA. The micro-lens and the end of the fiber cable is introduced inside the vacuum chamber through a gauge port and is made movable along the radius over $5 \mathrm{~cm}$ and along the $z$ axis over $30 \mathrm{~cm}$. The chamber has been evacuated down to $6 \times 10^{-6}$ Torr before the experiment.

\section{Results}

\subsection{Vacuum discharge in a transverse magnetic field}

Fig. 2 indicates the trigger characteristics of the plasma gun; the probability of stimulated vacuum breakdown between the anode $(A)$ and cathode $(K)$ is plotted as a function of $B_{z}$ and a capacitor charging voltage $V_{c}$ for a fixed triggering voltage of $10 \mathrm{kV}$. The inner diameter of the anode is $23 \mathrm{~mm}$ while the cathode has the diameter of $8 \mathrm{~mm}$ and is made of brass ( $\mathrm{Cu} 60 \%$ ) $\mathrm{Zn} 40 \%$ ), so that the $\mathrm{A}-\mathrm{K}$ gap spacing is $7.5 \mathrm{~mm}$. Five trigger sparks have been tested for each set of $B_{z}$ and $V_{c}$. The breakdown becomes more difficult to occur with increasing $B_{z}$ in the range $V_{c} \leq 7 \mathrm{kV}$. This is probably because the breakdown is promoted by the injection of charged particles and UV photons into the A-K gap while the charged particles are easily blocked by the transverse field $B_{z}$.

It is found that the magnetic field $B_{z}$ which traverses the annular $\mathrm{A}-\mathrm{K}$ gap plays a role of stabilizing vacuum discharge as well as driving the plasma rotation. Typical oscillograms of the diode voltage taken at $V_{c}=$ $9 \mathrm{kV}$ and at different $B_{z}$ are given in Fig. 3. Data are for the brass cathode but similar results have been obtained for $\mathrm{Cu}, \mathrm{Al}, \mathrm{Ag}$ and Ta cathodes. The lower trace shows

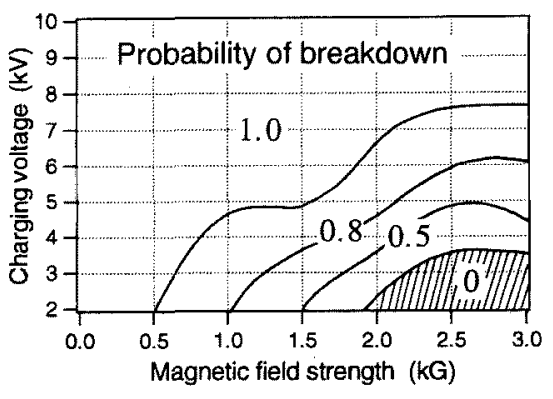

Fig. 2. Trigger characteristics of the plasma gun.

the diode current detected by the Rogowski coil. The diode current rises to a peak value of $13 \mathrm{kA}\left(V_{c}=9 \mathrm{kV}\right)$ in $8 \mu \mathrm{s}$ and falls to zero in $30 \mu \mathrm{s}$; the waveform is the same for different $B_{z}$ because the effective plasma impedance of $0.02 \Omega$ is much smaller than the driver circuit impedance of $0.67 \Omega$. For $B_{z}=2$ and $3 \mathrm{kG}$, waveforms are quite smooth and reproducible and any difference can not be identified between them. On the other hand, the fluctuation of the voltage at about 1 $\mathrm{MHz}$ grows rapidly with decreasing $B_{z}$ to less than $1.5 \mathrm{kG}$ and the relative voltage fluctuation $\tilde{V}_{d} / V_{d}$ reaches as high as $100 \%$ at $B_{z}=0$, implying destabilization of the discharge. The shot-to-shot variation in the waveform becomes quite large in this range.

Fig. 4 shows typical examples of end-on framing photographs. When $B_{z}=2.5 \mathrm{kG}$ is applied, the vacuum discharge which first started at a local position on the cathode expands over the whole gap in a time of about $1 \mu \mathrm{s}$ with increasing its luminosity and appears to form an annular glow region surrounding the cathode surface. This observation suggests that electrons are accelerated by the cathode fall up to the energy $e V_{c} \approx e V_{d}$ and the anode fall is negligible, so that the ionization of the metal vapor occurs predominantly near the cathode surface. On the other hand, the discharge looks very 


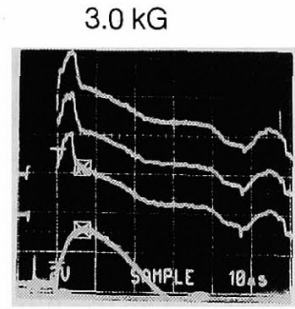

$0.5 \mathrm{kG}$

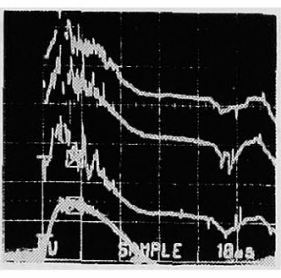

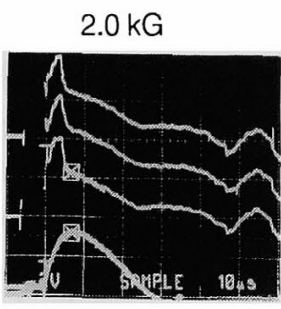

$0.1 \mathrm{kG}$

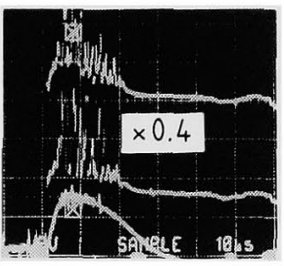

$1.5 \mathrm{kG}$

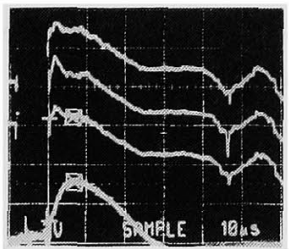

Upper: Voltage $250 \mathrm{~V} / \mathrm{div}$

Lower: Current $5.3 \mathrm{kA} / \mathrm{div}$

Fig. 3. Waveforms of the diode voltage at different $B_{z}$.
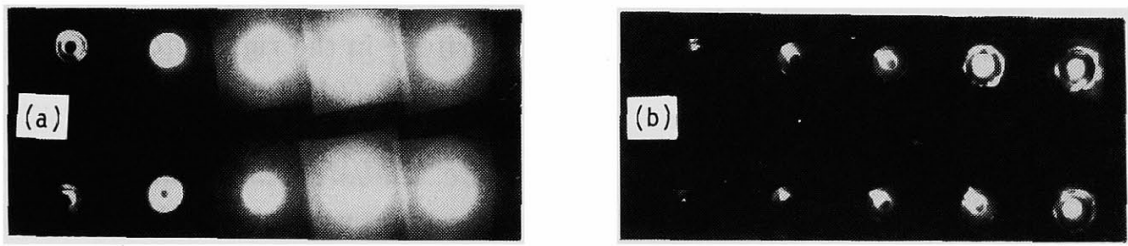

Fig. 4. End-on framing photographs of the vacuum discharge for (a) $B_{z}=2.5 \mathrm{kG}$ and ( $\mathrm{b}$ ) $B_{z}=0 \mathrm{kG}$. The sequence is from the bottom left to the top right. The framing speed is $0.5 \mu \mathrm{s} /$ frame.

(a)

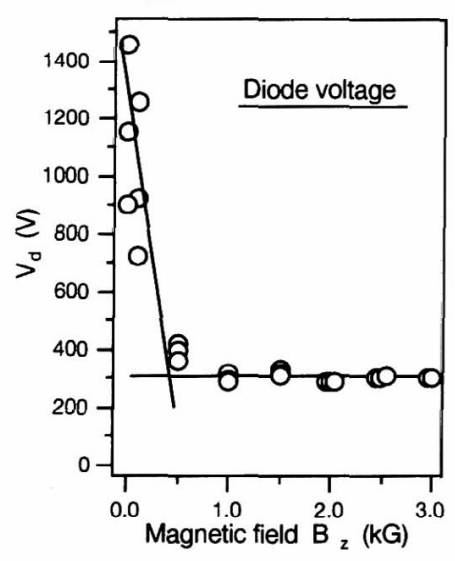

(b)

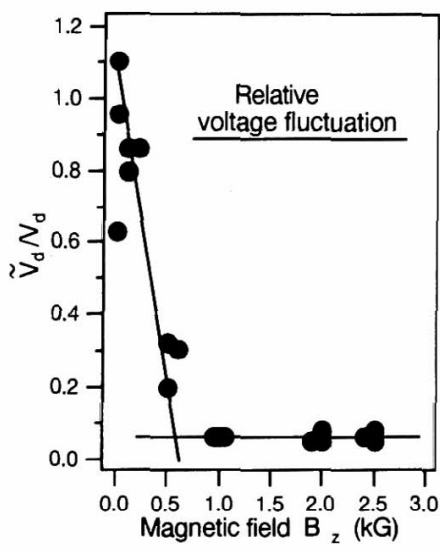

(c)

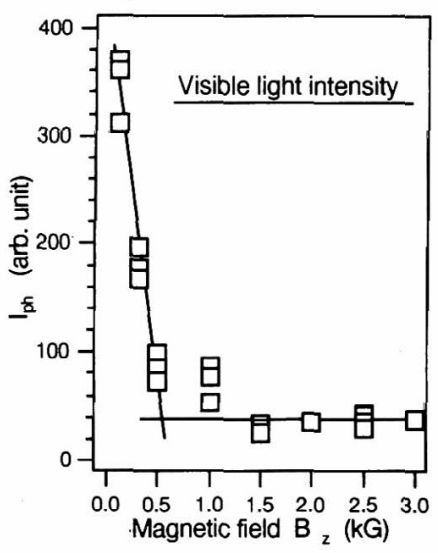

Fig. 5. Diode voltage, relative voltage fluctuation and visible light intensity as a function of $B_{z}$.

irregular at $B_{z}=0$ as demonstrated by some pieces of moving spokes. These behaviors are well correlated with voltage waveforms in Fig. 3.

In Fig. 5, (a) the diode voltage $V_{d}$ (DC component), (b) the relative voltage fluctuation $\tilde{V}_{d} / V_{d}$ and (c) the visible light intensity $I_{p h}$ detected by a PIN diode are plotted as a function of $B_{z}$. These data demonstrate the existence of a critical magnetic field strength $B_{c} \approx 0.3$ $\mathrm{kG}$ for stabilizing the intense vacuum discharge: In the range $B_{z}>B_{c}, V_{d}$ is kept constant at about $280 \mathrm{~V}$ independent of $B_{z}$ and the voltage fluctuation $V_{d} / V_{d}$ is negligibly small there, while $B_{z}<B_{c}, V_{d}$ rapidly increases up to $1,000 \mathrm{~V}$ with decreasing $B_{z}$ to $0 \mathrm{G}$ and $V_{d} / V_{d}$ increases up to about 1.0 in the same manner. This clearly indicates that a transverse magnetic field in excess of a critical strength is essential for stabilizing the intense vacuum discharge and for producing the dense metal plasma efficiently. Note that $I_{p h}$ shows the same $B_{z}$ dependence as $V_{d}$. This result is also reasonable because $I_{p h}$ should be proportional to the plasma 
density $n$ while $n \propto V_{d} I_{d} \propto V_{d}$ for $I_{d}$ fixed at $13 \mathrm{kA}$. A model to derive $B_{c}$ will be discussed in the next chapter.

\subsection{Production of rotating plasmas}

Rotating metal plasmas have been produced with brass and Al cathodes to explore the isotope separation by the plasma centrifuge. The plasma rotation has been driven by the $\boldsymbol{J} \times \boldsymbol{B}$ Lorentz force where $\boldsymbol{J}$ is the radial current density of the discharge and $B$ is the applied field. Since $J$ produces an azimuthal magnetic field $B_{\theta}$, the plasma also is accelerated outward along the axis as is the case of conventional, coaxial-plasma-accelerators.

Typical plasma parameters for $\mathrm{Cu} / \mathrm{Zn}$ plasmas ${ }^{(9)}\left(I_{d}=\right.$ $13 \mathrm{kA} / B_{z}=2.5 \mathrm{kG}$ ) are: The electron temperature remains $10 \sim 12 \mathrm{eV}$ independent of $I_{d}$ and $B_{z}$. The ion temperature is in the range $10 \sim 30 \mathrm{eV}$. The plasma density is the order of $10^{15} \mathrm{~cm}^{-3}$ (single-charged ions are assumed) and further increases in proportion to $I_{d}^{2}$ since $V_{d} \propto I_{d}$ experimentally. The axial flow velocity is about $2 \times 10^{4} \mathrm{~m} / \mathrm{s}$.

Emission spectra have been measured by OMA over the wavelength from 290 to $610 \mathrm{~nm}$. An example for the $\mathrm{Cu} / \mathrm{Zn}$ plasma is given in Fig. 6 where the wavelength is from 489 to $509 \mathrm{~nm}$. The detection of impurities, $C$ and $O$ implies that they have been sputtered from polymer and glass surfaces inside the plasma gun by the

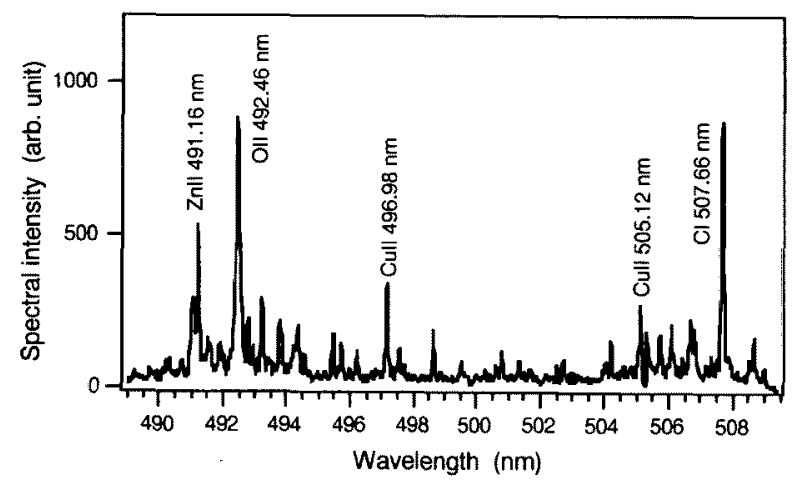

Fig. 6. Emission spectra of the $\mathrm{Cu} / \mathrm{Zn}$ plasma.

(a) Znll $491.16 \mathrm{~nm}$

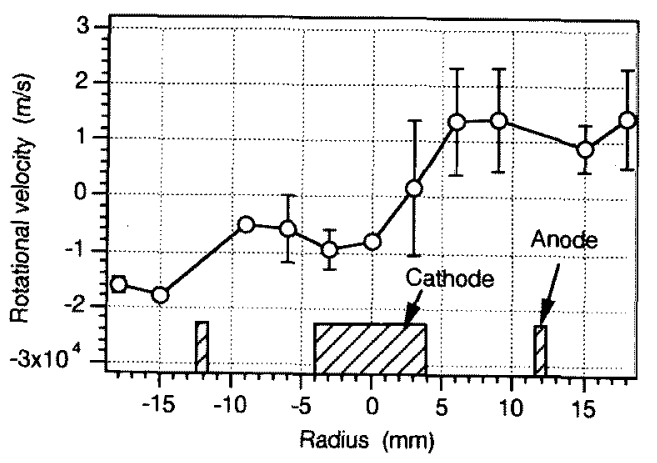

bombardment of $\mathrm{Cu}^{+}$and $\mathrm{Zn}^{+}$ions. Since spectral intensities of CuIl and $\mathrm{ZnII}$ are superior in the UV range $(<300$ $\mathrm{nm})$, they appear rather weak compared with $\mathrm{CII}$ and OII in this visible range. Representative two lines, $\mathrm{ZnII}$ $491.16 \mathrm{~nm}$ and OII $492.46 \mathrm{~nm}$ have been chosen to measure profiles of the rotational velocity from the spectroscopic doppler shift. The results are given in Fig. 7. Error bars on data points show shot-to-shot variations. We will derive the following features from the figure:

(1) Roughly speaking, velocity profiles for $\mathrm{Zn}^{+}$and $\mathrm{O}^{+}$ions are the same.

(2) The plasma core within the diameter of about $1 \mathrm{~cm}$ is in rigid rotation at $\omega \approx 2.5 \times 10^{6} \mathrm{rad} / \mathrm{s}$.

(3) On the other hand, the plasma outside rotates at a constant velocity of about $1 \times 10^{4} \mathrm{~m} / \mathrm{s}$; that is, a shear flow is formed around the plasma core.

The mass separation factor $\alpha$ for the plasma centrifuge in the rigid-rotor equilibrium is given by

$$
\alpha=\exp \left\{\left(\frac{\Delta M}{M}\right) \Theta\right\}
$$

where, $\Delta M / M=2\left(M_{H}-M_{L}\right) /\left(M_{H}+M_{L}\right)$ is the relative mass difference between heavier $\left(M_{H}\right)$ and lighter $\left(M_{L}\right)$ ion species and $\Theta=M r^{2} \omega^{2} / 2 T_{i}$. The rotation frequency observed here is quite large compared with those in earlier experiments ${ }^{(5)}$. Thus, a considerable improvement in the separation factor can be expected according to Eq. (1)

\section{Discussion}

Here, we derive the critical field strength $B_{c}$ for stabilizing the vacuum discharge from a simple consideration. Suppose a coaxial diode with the anode radius $r_{a}$, the cathode radius $r_{k}$ and the AK spacing $d \equiv r_{a}-r_{k}$ as shown in Fig. 8. An electron from the cathode is accelerated in the positive sheath in front of the cathode ( $d_{s}$ in thickness) up to an energy $e V_{c}$ and enters the plasma-filled gap under the uniform $B_{z}$. The diode

(b) Oll $492.46 \mathrm{~nm}$

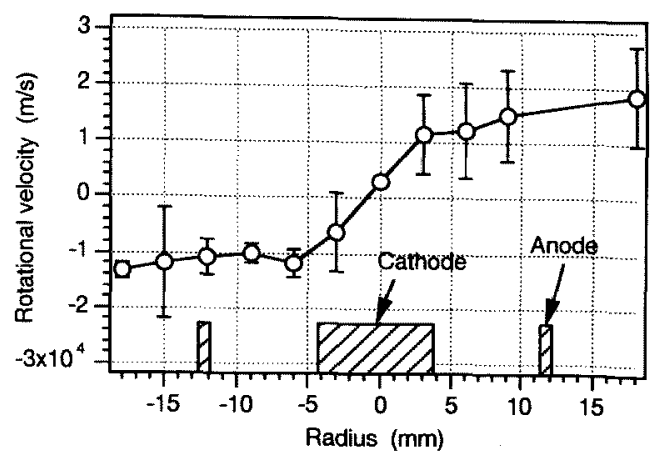

Fig. 7. Rotational velocity profiles for (a) $\mathrm{Zn}^{+}$and (b) $\mathrm{O}^{+}$ions. 
Bz

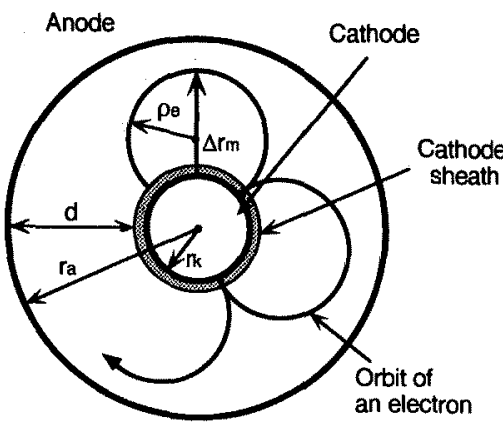

Fig. 8. Orbit of an electron in the diode gap.

voltage can be expressed as $V_{d}=V_{c}+\Delta V$ and from measurements can be approximated to $V_{d} \approx V_{c}$, where $\Delta V$ denotes the potential drop due to the plasma rotation, $\left(r_{a}^{2}-r_{k}^{2}\right) \omega B_{z} / 2$. The sheath potential due to the uniform ion space-charge of density $n$ is given by

$$
V_{c}=\frac{e n}{2 \varepsilon_{0}} d_{s}^{2} \text {. }
$$

The sheath is very thin since $d_{s} \approx 4.7 \times 10^{-3} \mathrm{~mm} \ll d=7.5$ $\mathrm{mm}$ is calculated from measured values, $n \sim 10^{15} \mathrm{~cm}^{-3}$ and $V_{c} \approx 200 \mathrm{~V}$. The electron of mass $m$ first moves toward the anode along a circular orbit of radius

$$
\rho_{e}=\sqrt{2 m V_{c} / e B_{z}^{2}}
$$

If a maximum traveling distance, $\Delta r_{m}<d$, the electron turns at $\Delta r_{m}$ and re-enters the sheath at right angle to the surface, where it is again reflected back to the anode. As a result, the electron continues to drift around the axis until it is scattered by collision with plasma ions, plasma electrons and neutral atoms as illustrated in Fig. 8. On the other hand, if $\Delta r_{m}>d$, the electron is lost to the anode in a time shorter than the electron gyroperiod, $2 \pi \Omega_{e}^{-1}$. The good electron confinement will improve the efficiency and uniformity of ionization of vapor atoms and will result in a considerable reduction in the diode voltage. Here, we have assumed $\Omega_{e} \tau_{e} \gg 1$ reasonably where $\tau_{e}$ is the electron collision time. This assumption is valid because $\Omega_{e} \tau_{e} \approx \Omega_{e} \tau_{e n} \sim 10 \gg 1$ has been estimated from experimental parameters, where $\tau_{e n}$ is the electronneutral collision time. If $\Omega_{e} \tau_{e} \ll 1$ was the case, the effect of $B_{z}$ would not appear or would be obscure in the experimental results.

From Fig. 8. $\Delta r_{m}$ is given by

$$
\Delta r_{m}=\sqrt{\rho_{e}^{2}+r_{k}^{2}}+\rho_{e}-r_{k}
$$

The critical strength $B_{c}$ for the stable discharge is therefore derived by solving the equation $\Delta r_{m}=d$ as

$$
B_{c}=\left(\frac{1+\delta}{1+2 \delta}\right) \sqrt{\frac{8 m V_{c}}{e d^{2}}}
$$

where $\delta \equiv \gamma_{k} / d$.

Fig. 9 shows $V_{c}$ as a function of $B_{z}$ measured, together

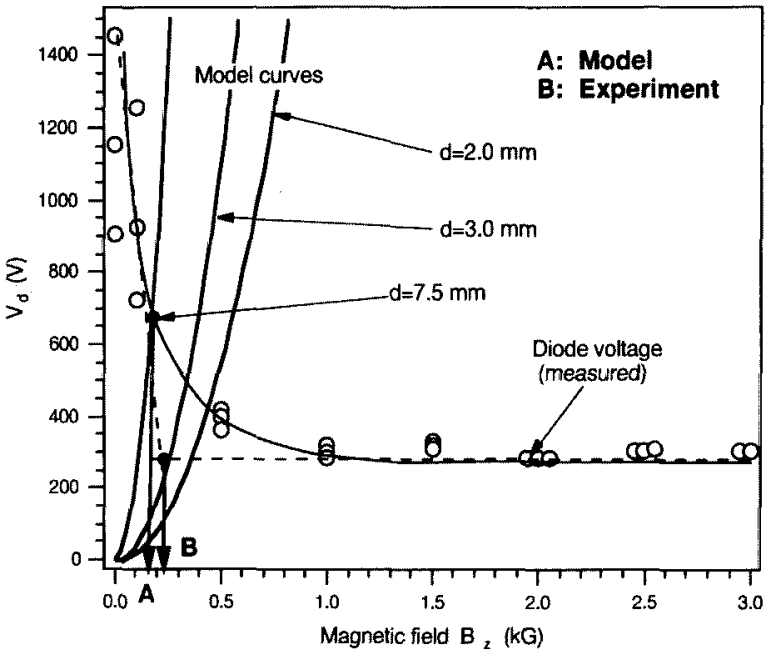

Fig. 9. Derivation of the critical field strength.

with the theoretical $V_{c}-B_{c}$ curve [Eq. ( 5 )] for $d=7.5$ $\mathrm{mm}$ and $\delta=0.53$ of the present experiment. The theoretical value, $B_{c}$ (model) is evaluated from the intersection $A$ between two curves. This yields $B_{c}$ (model) $\approx 170$ $\mathrm{G}$. On the other hand, the inflection point $B$ on the $V_{c}$ $B_{z}$ data curve will give $B_{c}(\exp ) \approx 250 \mathrm{G}$. Agreement between $B_{c}(\exp )$ and $B_{c}$ (model) seems good. Comparison between $B_{c}(\exp )$ and $B_{c}$ (model) has been carried out for $I_{d}=7$ and $13 \mathrm{kA}$ and $d=3.0$ and $7.5 \mathrm{~mm}$; the difference was within a factor of 2 . Thus, the present model is sufficient to explain the observation.

\section{Conclusion}

The stabilizing effect of a transverse magnetic field $B_{z}$ has been investigated on the intense vacuum discharge of the coaxial plasma gun which has been developed to generate rotating metal plasmas for the plasma centrifuge. In typical, the experiment was carried out at the diode current $I_{d}$ of $13 \mathrm{kA}$ (20 $\mu$ s duration) and $B_{z}$ of up to $3.0 \mathrm{kG}$. A quite stable and uniform discharge was found to be established even in the high power when $B_{z}$ in excess of a critical value of $250 \mathrm{G}$ was applied; otherwise, the discharge became unstable. A model which considers the trapping of ionizing, beam electrons in the annular A-K gap was found to predict the critical field strength qualitatively.

Rotating $\mathrm{Cu} / \mathrm{Zn}$ plasmas have been produced at $I_{\alpha}=13$ $\mathrm{kA}$ and $B_{z}=2.5 \mathrm{kG}$. Spectroscopic doppler measurements revealed that $\mathrm{Zn}^{+}$ions within the core region of a diameter of $1 \mathrm{~cm}$ rotate rigidly, while the velocity of them appears to be saturated outside. The plasma density was the order of $10^{15} \mathrm{~cm}^{-3}$ and the rotation frequency was about $2.5 \times 10^{6} \mathrm{rad} / \mathrm{s}$.

(Manuscript received July 12, '93, revised Oct. 27, '93) 


\section{References}

(1) D.G. Beanland: Ion Implantation and Beam Processing (1984) Academic Press, Sydney

(2) I. I. Aksenov, S. I. Vakula, V. G. Padalka, V. E. Strelnitskii \& V. M. Khoroshikh: Sov. Phys. Tech. Phys., 25, 1164 (1980)

(3) L. K. Len, C. Burkhart, G. W. Cooper, S. Humphries, Jr., M. Savage \& D. M. Woodall : IEEE Trans. Plasma Sci., PS-14, 256 (1986)

(4) P. Louvet: Proc. 2nd Workshop on Separation Phenomena in Liquids and Gases, Versailles, p. 5 (1989)

(5) M. Geva, M. Krishnan \& J. L. Hirshfield : J. Appl. Phys., 56, 1398 (1984)

(6) R. R. Prasad \& M. Krishnan: ibid., 61, 4464 (1987)

(7) I. G. Brown: IEEE Trans. Plasma Sci., PS-15, 346 (1987)

(8) C. W. Kimblin: ibid., PS-10, 322 (1982)

(9) T. Ikehata, K. Oohashi, N. Y. Sato, T. Tanabe \& H. Mase: Nucl. Instrum. Method, B70, 26 (1992)

Takashi Ikehata (Member) He was born in 1954 and received

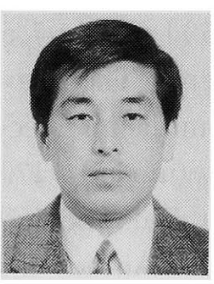
Ph. D. degree in physics from Tsukuba University in 1983. Since 1983 he has been in the Department of Electrical and Electronic Engineering, Ibaraki University and is currently an Associate Professor. His research interest has been in the physics and applications of plasmas such as intense plasma beams, rotat ing plasmas for plasma centrifuge and processing plasmas.

Dr. Ikehata is a member the Physical Society of Japan, the Japan Society of Applied Physics and the Japan Society of Plasma Science and Nuclear Fusion Research.

Kazuyuki Hasegawa (Non-member) He was born in 1967 and

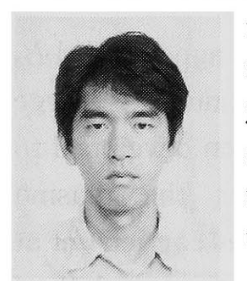
received the M.S. degree in Electronic Engineering from Ibaraki University in 1991. He joined Fuji Photo Film Co. Ltd. in 1991 and since then he has been engaged in the development of MiniLabo photographic equipments at the Miyanodai Development Center.
Toshio Tanabe (Non-member) He was born in 1943 and

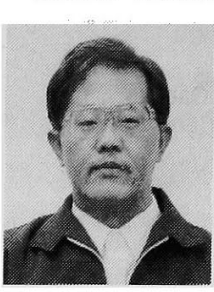
graduated Ibaraki University in 1970. Since 1965 he has been in the Department of Electrical and Electronic Engineering, Ibaraki University as a technical stuff and has been engaged in the development of plasma processing devices and the study on plasma applications.

Naoyuki Sato (Non-member) He was born in 1962 and

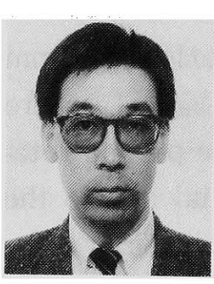
received the $\mathrm{Ph}$. D. degree in Electronic Engineering from Tohoku University in 1990. Since 1990 he has been in the Department of Electrical and Electronic Engineering, Ibaraki University and is currently a research associate. He has studied the interactions between charged particles and waves in plasmas.

Dr. Sato is a member of the Physical Society of Japan and the Japan Society of Plasma Science and Nuclear Fusion Research.

Hiroshi Mase (Member) He was born in 1936 and received

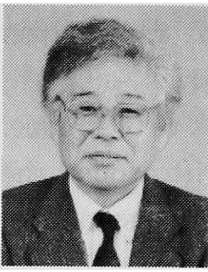
the $\mathrm{Ph}$. D. degree in Electrical Engineering from Tohoku University in 1965. Since 1966 he has been in the Department of Electrical and Electronic Engineering, Ibaraki University and is currently a Professor. His research includes the modeling of electric discharges, the development of plasma sources, plasma processing and the physics and applications of rotating plasmas.

Dr. Mase is a member of the Physical Society of Japan, the Japan Society of Applied Physics, the Japan Society of Plasma Science and Nuclear Fusion Research and the American Physical Society. 